\title{
Stability for Functional Equation of Mixed Type in Non-Archimedean Normed Spaces
}

\author{
Ick-Soon Chang ${ }^{1}$ and Yang-Hi Lee ${ }^{2}$ \\ ${ }^{1}$ Department of Mathematics, Chungnam National University, 79 Daehangno, Yuseong-gu, Daejeon 305-764, Republic of Korea \\ ${ }^{2}$ Department of Mathematics Education, Gongju National University of Education, Gongju 314-711, Republic of Korea \\ Correspondence should be addressed to Ick-Soon Chang; ischang@cnu.ac.kr
}

Received 16 November 2013; Revised 8 January 2014; Accepted 10 January 2014; Published 25 February 2014

Academic Editor: Krzysztof Ciepliński

Copyright (C) 2014 I.-S. Chang and Y.-H. Lee. This is an open access article distributed under the Creative Commons Attribution License, which permits unrestricted use, distribution, and reproduction in any medium, provided the original work is properly cited.

We take into account the stability of the following functional equation $f(x+a y)+f(x-a y)-2 f(x)-a^{2} f(y)-a^{2} f(-y)=0$ in non-Archimedean normed spaces.

\section{Introduction and Preliminaries}

The study of stability problems has originally been formulated by Ulam [1]: under what condition does there exist a homomorphism near an approximate homomorphism? Hyers [2] had answered affirmatively the question of Ulam for Banach spaces. The theorem of Hyers was generalized by Aoki [3] for additive mappings and by Rassias [4] for linear mappings by considering an unbounded Cauchy difference. The paper work of Rassias [4] has had a lot of influence in the development of what is called the generalized Hyers-Ulam stability of functional equations. Thereafter, many interesting results of the generalized Hyers-Ulam stability to a number of functional equations have been investigated by a number of mathematicians; see [5-13] and references therein.

Now we demonstrate some definitions used in this work.

Definition 1. A field $\mathbb{K}$, equipped with a function (valuation) $|\cdot|$ from $\mathbb{K}$ into $[0, \infty)$, is called a non-Archimedean field if the function $|\cdot|: \mathbb{K} \rightarrow[0, \infty)$ satisfies the following conditions:

(1) $|r|=0$ if and only if $r=0$;

(2) $|r s|=|r||s|$;

(3) the strong triangle inequality, namely, $|r+s| \leq$ $\max \{|r|,|s|\}$ for all $r, s \in \mathbb{K}$.

Of course, it is easy to see that $|1|=1=|-1|$ and $|n| \leq 1$ for all nonzero integer $n$.
Definition 2. Let $\mathscr{X}$ be a vector space over the nonArchimedean field $\mathbb{K}$ with a nontrivial non-Archimedean valuation $|\cdot|$. A function $\|\cdot\|: \mathscr{X} \rightarrow[0, \infty)$ is said to be a non-Archimedean norm (valuation) if it satisfies the following conditions:

(1) $\|x\|=0$ if and only if $x=0$;

(2) $\|r x\|=|r|\|x\|$ for all $x \in \mathscr{X}$ and all $r \in \mathbb{K}$;

(3) the strong triangle inequality, namely,

$$
\|x+y\| \leq \max \{\|x\|,\|y\|\} \quad \forall x, y \in \mathscr{X} .
$$

In this case, $(\mathscr{X},\|\cdot\|)$ is called a non-Archimedean space. Moreover, if every Cauchy sequence is convergent, then $(\mathscr{X}$, $\|$. $\|)$ is said to be a complete non-Archimedean space.

It follows from the strong triangle inequality that

$$
\left\|x_{n}-x_{m}\right\| \leq \max \left\{\left\|x_{j+1}-x_{j}\right\|: m \leq j<n-1\right\},
$$

for all $x_{n}, x_{m} \in \mathscr{X}$ and all $m, n \in \mathbb{N}$ with $n>m$. Therefore a sequence $\left\{x_{n}\right\}$ is a Cauchy sequence in non-Archimedean space if and only if the sequence $\left\{x_{n+1}-x_{n}\right\}$ converges to zero in the space.

On the other hand, Moslehian and Rassias [14] discussed the stability of the additive functional equation and the quadratic functional equation in non-Archimedean normed 
spaces. Quite recently, the new results on stability of functional equations in non-Archimedean metric spaces have been investigated (e.g., $[6,12,15])$.

Here and now, we consider a quadratic-additive type functional equation

$$
f(x+a y)+f(x-a y)-2 f(x)-a^{2} f(y)-a^{2} f(-y)=0,
$$

whose solution is called a quadratic-additive mapping. Quite recently, the second author [16] investigated the HyersUlam stability of the functional equation (3) on restricted domains for the case $a=1$. In particular, hyperstability of the functional equation (3) in the case when $a=1$ was investigated in [17]. The purpose of this work establishes the generalized Hyers-Ulam stability of the functional equation (3) in non-Archimedean normed spaces.

\section{Main Results}

Throughout this section, we assume that $\mathscr{X}$ is a nonArchimedean normed space and $\mathcal{Y}$ is a complete nonArchimedean space. For a given mapping $f: E_{1} \rightarrow E_{2}$ with vector spaces $E_{1}$ and $E_{2}$, we use the abbreviations

$$
\begin{gathered}
\mathscr{A} f(x, y):=f(x+y)-f(x)-f(y), \\
\mathcal{Q} f(x, y):=f(x+y)+f(x-y)-2 f(x)-2 f(y), \\
\operatorname{Df}(x, y):=f(x+a y)+f(x-a y) \\
-2 f(x)-a^{2} f(y)-a^{2} f(-y)
\end{gathered}
$$

for all $x, y \in E_{1}$, where $a$ is a nonzero integer.

Theorem 3. Let $E_{1}$ and $E_{2}$ be vector spaces. A mapping $f$ : $E_{1} \rightarrow E_{2}$ satisfies equation $D f(x, y)=0$ for all $x, y \in E_{1}$ if and only if there exist a quadratic mapping $g: E_{1} \rightarrow E_{2}$ and an additive mapping $h: E_{1} \rightarrow E_{2}$ such that

$$
f(x)=g(x)+h(x),
$$

for all $x \in E_{1}$.

Proof. $(\Rightarrow)$ We decompose $f$ into the even part and the odd part by putting

$$
\begin{aligned}
& g(x)=\frac{f(x)+f(-x)}{2}, \\
& h(x)=\frac{f(x)-f(-x)}{2},
\end{aligned}
$$

for all $x \in E_{1}$. Notice that $f(0)=-D f(0,0) / 2 a^{2}=0$. From the equalities

$$
\begin{gathered}
Q g \\
Q \\
\mathscr{A} h(x, y)=\operatorname{Dg}\left(x, \frac{y}{a}\right)-\operatorname{Dg}\left(0, \frac{y}{a}\right)=0, \\
D h\left(\frac{x+y}{2}, \frac{x+y}{2 a}\right)-\operatorname{Dh}\left(\frac{x+y}{2}, \frac{x-y}{2 a}\right)=0,
\end{gathered}
$$

for all $x, y \in E_{1}$, we conclude that $g$ is a quadratic mapping and $h$ is an additive mapping.

$(\Leftarrow)$ If there exist a quadratic mapping $g: E_{1} \rightarrow E_{2}$ and an additive mapping $h: E_{1} \rightarrow E_{2}$ such that

$$
f(x)=g(x)+h(x)
$$

for all $x \in E_{1}$, then we find that

$$
\begin{aligned}
\operatorname{Df}(x, y)= & \operatorname{Dg}(x, y)+\operatorname{Dh}(x, y) \\
= & \mathscr{Q} g(x, a y)+2 g(a y)-a^{2} g(y)-a^{2} g(-y) \\
& +\mathscr{A} h(x, x)-\mathscr{A} h(x+a y, x-a y) \\
= & 0,
\end{aligned}
$$

for all $x, y \in E_{1}$. We arrive at the desired conclusion.

Theorem 4. Let $\varphi: \mathscr{X}^{2} \rightarrow[0, \infty)$ be a function such that

$$
\lim _{n \rightarrow \infty} \frac{\varphi\left(2^{n} x, 2^{n} y\right)}{|2|^{n}}=\frac{\varphi\left(a^{n} x, a^{n} y\right)}{|a|^{2 n}}=0
$$

for all $x, y \in \mathscr{X}$ and, for each $x \in \mathscr{X}$, let the limit

$$
\begin{aligned}
\lim _{n \rightarrow \infty} \max \left\{\frac{\varphi\left(0, a^{j} x\right)}{|2||a|^{2 j+2}}, \frac{\varphi\left(2^{j} x, 2^{j} x / a\right)}{|2|^{j+2}},\right. \\
\left.\frac{\varphi\left(-2^{j} x,-2^{j} x / a\right)}{|2|^{j+2}}: 0 \leq j<n\right\},
\end{aligned}
$$

denoted by $\widetilde{\varphi}(x)$, exist. Suppose that $f: \mathscr{X} \rightarrow \mathcal{Y}$ is a mapping satisfying

$$
\|D f(x, y)\| \leq \varphi(x, y)
$$

for all $x, y \in \mathscr{X}$. Then there exists a quadratic-additive mapping $T: X \rightarrow \mathscr{Y}$ such that

$$
\|f(x)-T(x)\| \leq \widetilde{\varphi}(x),
$$

for all $x \in \mathscr{X}$, where the mapping $T$ is given by

$$
T(x)=\lim _{n \rightarrow \infty}\left[\frac{f\left(a^{n} x\right)+f\left(-a^{n} x\right)}{2 a^{2 n}}+\frac{f\left(2^{n} x\right)-f\left(-2^{n} x\right)}{2^{n+1}}\right],
$$

for all $x \in \mathscr{X}$.

Proof. For a given mapping $f: \mathscr{X} \rightarrow \mathscr{Y}$ and $n \in \mathbb{N}$, let $J_{n} f: \mathscr{X} \rightarrow \mathcal{Y}$ be a mapping defined by

$$
J_{n} f(x)=\frac{f\left(a^{n} x\right)+f\left(-a^{n} x\right)}{2 a^{2 n}}+\frac{f\left(2^{n} x\right)-f\left(-2^{n} x\right)}{2^{n+1}},
$$


for all $x \in \mathscr{X}$. Note that $J_{0} f(x)=f(x)$ and

$$
\begin{aligned}
\left\|J_{j} f(x)-J_{j+1} f(x)\right\|= & -\frac{D f\left(0, a^{j} x\right)}{2 a^{2 j+2}-\frac{D f\left(2^{j} x, 2^{j} x / a\right)}{2^{j+2}}} \\
& +\frac{D f\left(-2^{j} x,-2^{j} x / a\right) \|}{2^{j+2}} \| \\
\leq & \max \left\{\frac{\left\|D f\left(0, a^{j} x\right)\right\|}{|2||a|^{2 j+2}},\right. \\
& \frac{\left\|D f\left(2^{j} x, 2^{j} x / a\right)\right\|}{|2|^{j+2}}, \\
\leq \max \left\{\frac{\varphi\left(-2^{j} x,-2^{j} x / a\right) \|}{|2|^{j+2}}\right\} & \frac{\varphi\left(-a^{j} x\right)}{\left.|2||a|^{2 j+2}, \frac{\varphi\left(2^{j} x, 2^{j} x / a\right)}{|2|^{j+2}} \mid 2^{j+2} x / a\right)},
\end{aligned}
$$

for all $x \in \mathscr{X}$ and $j \geq 0$. It follows from (10) and (16) that the sequence $\left\{J_{n} f(x)\right\}$ is Cauchy. Since $\mathcal{Y}$ is complete, we conclude that $\left\{J_{n} f(x)\right\}$ is convergent. So we can define $T: X \rightarrow \mathscr{Y}$ by

$$
T(x):=\lim _{n \rightarrow \infty} J_{n} f(x),
$$

for all $x \in \mathscr{X}$. An induction implies that

$$
\begin{aligned}
& \left\|J_{n} f(x)-f(x)\right\| \leq \max \left\{\frac{\varphi\left(0, a^{j} x\right)}{|2||a|^{2 j+2}}, \frac{\varphi\left(2^{j} x, 2^{j} x / a\right)}{|2|^{j+2}},\right. \\
& \left.\frac{\varphi\left(-2^{j} x,-2^{j} x / a\right)}{|2|^{j+2}}: 0 \leq j<n\right\},
\end{aligned}
$$

for all $n \in \mathbb{N}$ and all $x \in \mathscr{X}$. By taking the limit as $n \rightarrow \infty$ in (18) and using (10), we obtain inequality (13).

From (12), we get

$$
\begin{aligned}
&\left\|D J_{n} f(x, y)\right\|=\| \frac{D f\left(2^{n} x, 2^{n} y\right)-D f\left(-2^{n} x,-2^{n} y\right)}{2^{n+1}} \\
&+\frac{D f\left(a^{n} x, a^{n} y\right)+D f\left(-a^{n} x,-a^{n} y\right)}{2 a^{2 n}} \| \max \left\{\frac{\varphi\left(2^{n} x, 2^{n} y\right)}{|2|^{n+1}}, \frac{\varphi\left(-2^{n} x,-2^{n} y\right)}{|2|^{n+1}},\right. \\
&\left.\frac{\varphi\left(a^{n} x, a^{n} y\right)}{|2||a|^{2 n}}, \frac{\varphi\left(-a^{n} x,-a^{n} y\right)}{|2||a|^{2 n}}\right\}
\end{aligned}
$$

for all $x, y \in \mathscr{X}$. Take the limit as $n \rightarrow \infty$ in the above inequality and then use (10) to have $\operatorname{DT}(x, y)=0$ for all $x, y \in \mathscr{X}$.

Corollary 5. Let $r>2$ be a real number and $|2|,|a|<1$. If a mapping $f: \mathscr{X} \rightarrow \mathcal{Y}$ satisfies the condition

$$
\|D f(x, y)\| \leq \theta\left(\|x\|^{r}+\|y\|^{r}\right)
$$

for all $x, y \in \mathscr{X}$, then there exists a unique quadratic-additive mapping $T: \mathscr{X} \rightarrow \mathcal{Y}$ such that

$$
\|f(x)-T(x)\| \leq \frac{\left(|a|^{r}+1\right) \theta\|x\|^{r}}{|2|^{2}|a|^{r}},
$$

for all $x \in \mathscr{X}$.

Proof. Let $\varphi(x, y)=\theta\left(\|x\|^{r}+\|y\|^{r}\right)$. Since $|2|^{r-1}<1$ and $|a|^{r-2}<1$, we know that

$$
\lim _{n \rightarrow \infty} \frac{\varphi\left(2^{n} x, 2^{n} y\right)}{|2|^{n}}=\frac{\varphi\left(a^{n} x, a^{n} y\right)}{|a|^{2 n}}=0
$$

for all $x, y \in \mathscr{X}$. Therefore the conditions of Theorem 4 are fulfilled. In particular, it is easy to see that

$$
\begin{aligned}
\tilde{\varphi}(x) & =\max \left\{\frac{\theta\|x\|^{r}}{|2||a|^{2}}, \frac{\left(|a|^{r}+1\right) \theta\|x\|^{r}}{|2|^{2}|a|^{r}}\right\} \\
& =\frac{\left(|a|^{r}+1\right) \theta\|x\|^{r}}{|2|^{2}|a|^{r}} .
\end{aligned}
$$

Theorem 4 guarantees that there exists a quadratic-additive mapping $T: \mathscr{X} \rightarrow \mathcal{Y}$ with (21).

Now, to show uniqueness of the mapping $T$, let us assume that $T^{\prime}$ is another quadratic-additive mapping satisfying (21). Then we have

$$
\begin{aligned}
& T^{\prime}(x)-J_{k} T^{\prime}(x) \\
& =\sum_{j=0}^{k-1}\left(-\frac{D T^{\prime}\left(0, a^{j} x\right)}{2 a^{2 j+2}}\right. \\
& \left.\quad-\frac{D T^{\prime}\left(2^{j} x, 2^{j} x / a\right)}{2^{j+2}}+\frac{D T^{\prime}\left(-2^{j} x,-2^{j} x / a\right)}{2^{j+2}}\right) \\
& =0,
\end{aligned}
$$


for any $k \in \mathbb{N}$, and thus we feel that

$$
\begin{aligned}
& \left\|T(x)-T^{\prime}(x)\right\| \\
& =\lim _{k \rightarrow \infty}\left\|J_{k} T(x)-J_{k} T^{\prime}(x)\right\| \\
& \leq \lim _{k \rightarrow \infty} \max \left\{\left\|J_{k} T(x)-J_{k} f(x)\right\|,\right. \\
& \left.\quad\left\|J_{k} f(x)-J_{k} T^{\prime}(x)\right\|\right\} \\
& \leq \lim _{k \rightarrow \infty} \max \left\{|2|^{-k-1}\left\|T\left(2^{k} x\right)-f\left(2^{k} x\right)\right\|,\right. \\
& |2|^{-k-1}\left\|T\left(-2^{k} x\right)-f\left(-2^{k} x\right)\right\|, \\
& |2|^{-k-1}\left\|f\left(2^{k} x\right)-T^{\prime}\left(2^{k} x\right)\right\|, \\
& |2|^{-1}|a|^{-2 k}\left\|T\left(a^{k} x\right)-f\left(a^{k} x\right)\right\|, \\
& |2|^{-1}|a|^{-2 k}\left\|T\left(-a^{k} x\right)-f\left(-a^{k} x\right)\right\|, \\
& |2|^{-1}|a|^{-2 k}\left\|f\left(a^{k} x\right)-T^{\prime}\left(a^{k} x\right)\right\|, \\
& \left.|2|^{-1}|a|^{-2 k}\left\|f\left(-a^{k} x\right)-T^{\prime}\left(-a^{k} x\right)\right\|\right\} \\
& \left\{\begin{array}{l}
|a|^{k(r-2)}\left(|a|^{r}+1\right) \theta\|x\|^{r} \\
|2|^{3}|a|^{r}
\end{array},\right.
\end{aligned}
$$

for all $x \in \mathscr{X}$, which implies that $T$ is unique.

Theorem 6. Let $\varphi: X^{2} \rightarrow[0, \infty)$ be a function such that

$$
\lim _{n \rightarrow \infty}|2|^{n} \varphi\left(\frac{x}{2^{n}}, \frac{x}{2^{n}}\right)=\lim _{n \rightarrow \infty}|a|^{2 n} \varphi\left(\frac{x}{a^{n}}, \frac{y}{a^{n}}\right)=0
$$

for all $x, y \in \mathscr{X}$ and, for each $x \in \mathscr{X}$, let the limit

$$
\begin{gathered}
\lim _{n \rightarrow \infty} \max \left\{\frac{|a|^{2 j}}{|2|} \varphi\left(0, \frac{x}{a^{j+1}}\right),|2|^{j-1} \varphi\left(\frac{x}{2^{j+1}}, \frac{x}{2^{j+1} a}\right),\right. \\
\left.|2|^{j-1} \varphi\left(\frac{-x}{2^{j+1}}, \frac{-x}{2^{j+1} a}\right): 0 \leq j<n\right\},
\end{gathered}
$$

denoted by $\widetilde{\varphi}(x)$, exist. Suppose that $f: X \rightarrow \mathcal{Y}$ is a mapping satisfying

$$
\|D f(x, y)\| \leq \varphi(x, y)
$$

for all $x, y \in \mathscr{X}$. Then there exists a quadratic-additive mapping $T: \mathscr{X} \rightarrow \mathcal{Y}$ such that

$$
\|f(x)-T(x)\| \leq \widetilde{\varphi}(x),
$$

for all $x \in \mathscr{X}$, where the mapping $T$ is given by

$$
\begin{aligned}
T(x)=\lim _{n \rightarrow \infty}[ & \frac{a^{2 n}}{2}\left(f\left(\frac{x}{a^{n}}\right)+f\left(\frac{-x}{a^{n}}\right)\right) \\
& \left.+2^{n-1}\left(f\left(\frac{x}{2^{n}}\right)-f\left(\frac{-x}{2^{n}}\right)\right)\right],
\end{aligned}
$$

for all $x \in \mathscr{X}$.

Proof. For a given mapping $f: \mathscr{X} \rightarrow \mathcal{Y}$ and $n \in \mathbb{N}$, let $J_{n} f: \mathscr{X} \rightarrow \mathscr{Y}$ be a mapping defined by

$$
\begin{aligned}
J_{n} f(x)= & \frac{a^{2 n}}{2}\left(f\left(\frac{x}{a^{n}}\right)+f\left(\frac{-x}{a^{n}}\right)\right) \\
& +2^{n-1}\left(f\left(\frac{x}{2^{n}}\right)-f\left(\frac{-x}{2^{n}}\right)\right),
\end{aligned}
$$

for all $x \in \mathscr{X}$. Observe that $J_{0} f(x)=f(x)$ and

$$
\begin{aligned}
&\left\|J_{j} f(x)-J_{j+1} f(x)\right\|= \| \frac{a^{2 j}}{2} D f\left(0, \frac{x}{a^{j+1}}\right) \\
&+2^{j-1} D f\left(\frac{x}{2^{j+1}}, \frac{x}{2^{j+1} a}\right) \\
&-2^{j-1} D f\left(\frac{-x}{2^{j+1}}, \frac{-x}{2^{j+1} a}\right) \| \\
& \leq \max \left\{\frac{|a|^{2 j}}{|2|}\left\|D f\left(0, \frac{x}{a^{j+1}}\right)\right\|,\right. \\
&|2|^{j-1}\left\|D f\left(\frac{x}{2^{j+1}}, \frac{x}{2^{j+1} a}\right)\right\|,\left.|2|^{j-1}\left\|D f\left(\frac{-x}{2^{j+1}}, \frac{-x}{2^{j+1} a}\right)\right\|\right\} \\
& \leq \max \left\{\frac{|a|^{2 j}}{|2|} \varphi\left(0, \frac{x}{a^{j+1}}\right),\right. \\
&|2|^{j-1} \varphi\left(\frac{x}{2^{j+1}}, \frac{x}{2^{j+1} a}\right), \\
&\left.|2|^{j-1} \varphi\left(\frac{-x}{2^{j+1}}, \frac{-x}{2^{j+1} a}\right)\right\},
\end{aligned}
$$

for all $x \in \mathscr{X}$ and $j \geq 0$. It follows by (26) and (32) that the sequence $\left\{J_{n} f(x)\right\}$ is Cauchy. Since $\mathscr{Y}$ is complete, $\left\{J_{n} f(x)\right\}$ converges and so we can define $T: \mathscr{X} \rightarrow \mathcal{Y}$ by

$$
T(x):=\lim _{n \rightarrow \infty} J_{n} f(x)
$$


for all $x \in \mathscr{X}$. Applying the induction, we yield that

$$
\begin{gathered}
\left\|J_{n} f(x)-f(x)\right\| \\
\leq \max \left\{\frac{|a|^{2 j}}{|2|} \varphi\left(0, \frac{x}{a^{j+1}}\right),|2|^{j-1} \varphi\left(\frac{x}{2^{j+1}}, \frac{x}{2^{j+1} a}\right),\right. \\
\left.|2|^{j-1} \varphi\left(\frac{-x}{2^{j+1}}, \frac{-x}{2^{j+1} a}\right): 0 \leq j<n\right\},
\end{gathered}
$$

for all $n \in \mathbb{N}$ and all $x \in \mathscr{X}$. Sending the limit as $n \rightarrow \infty$ in (34) with (26), we arrive at inequality (29).

According to (28), we see that

$$
\begin{aligned}
& \left\|D J_{n} f(x, y)\right\| \\
& =\| \frac{a^{2 n}}{2}\left(D f\left(\frac{x}{a^{n}}, \frac{y}{a^{n}}\right)+D f\left(\frac{-x}{a^{n}}, \frac{-y}{a^{n}}\right)\right) \\
& +2^{n-1}\left(D f\left(\frac{x}{2^{n}}, \frac{y}{2^{n}}\right)-D f\left(\frac{-x}{2^{n}}, \frac{-y}{2^{n}}\right)\right) \| \\
& \leq \max \left\{\frac{|a|^{2 n}}{|2|} \varphi\left(\frac{x}{a^{n}}, \frac{y}{a^{n}}\right), \frac{|a|^{2 n}}{|2|} \varphi\left(\frac{-x}{a^{n}}, \frac{-y}{a^{n}}\right),\right. \\
& \left.|2|^{n-1} \varphi\left(\frac{x}{2^{n}}, \frac{y}{2^{n}}\right),|2|^{n-1} \varphi\left(\frac{-x}{2^{n}}, \frac{-y}{2^{n}}\right)\right\},
\end{aligned}
$$

for all $x, y \in \mathscr{X}$. Taking the limit as $n \rightarrow \infty$ and using (26), we get $D T(x, y)=0$ for all $x, y \in \mathscr{X}$.

Corollary 7. Let $r<1$ be a real number and $|2|,|a|<1$. If a mapping $f: X \rightarrow Y$ satisfies the condition

$$
\|D f(x, y)\| \leq \theta\left(\|x\|^{r}+\|y\|^{r}\right)
$$

for all $x, y \in \mathscr{X}$, then there exists a unique quadratic-additive mapping $T: \mathscr{X} \rightarrow \mathcal{Y}$ such that

$$
\|f(x)-T(x)\| \leq \frac{\left(|a|^{r}+1\right) \theta\|x\|^{r}}{|2|^{1+r}|a|^{r}}
$$

for all $x \in \mathscr{X}$.

Proof. Let $\varphi(x, y)=\theta\left(\|x\|^{r}+\|y\|^{r}\right)$. Since $|a|^{1-r}<1$ and $|a|^{2-r}<1$, the mapping $\varphi$ satisfies equalities (26) for all $x, y \in \mathscr{X}$. Moreover, we see that

$$
\tilde{\varphi}(x)=\max \left\{\frac{\theta\|x\|^{r}}{|2||a|^{r}}, \frac{\left(|a|^{r}+1\right) \theta\|x\|^{r}}{|2|^{1+r}|a|^{r}}\right\}=\frac{\left(|a|^{r}+1\right) \theta\|x\|^{r}}{|2|^{1+r}|a|^{r}} .
$$

Then it follows from Theorem 6 that there exists a quadraticadditive mapping $T: \mathscr{X} \rightarrow \mathscr{Y}$ satisfying (37).
In order to prove the uniqueness of $T$, we assume that $T^{\prime}$ is another quadratic-additive mapping satisfying (37). Then

$$
\begin{aligned}
T^{\prime}(x)-J_{k} T^{\prime}(x)=\sum_{j=0}^{k-1}\left(\frac{a^{2 j}}{2} D T^{\prime}\left(0, \frac{x}{a^{j+1}}\right)\right. \\
+2^{j-1} D T^{\prime}\left(\frac{x}{2^{j+1}}, \frac{x}{2^{j+1} a}\right) \\
\left.-2^{j-1} D T^{\prime}\left(\frac{-x}{2^{j+1}}, \frac{-x}{2^{j+1} a}\right)\right)=0,
\end{aligned}
$$

for any $k \in \mathbb{N}$, whence we give that

$$
\begin{aligned}
& \left\|T(x)-T^{\prime}(x)\right\| \\
& =\lim _{k \rightarrow \infty}\left\|J_{k} T(x)-J_{k} T^{\prime}(x)\right\| \\
& \leq \lim _{k \rightarrow \infty} \max \left\{\left\|J_{k} T(x)-J_{k} f(x)\right\|,\left\|J_{k} f(x)-J_{k} T^{\prime}(x)\right\|\right\} \\
& \leq \lim _{k \rightarrow \infty} \max \left\{|2|^{k-1}\left\|(T-f)\left(\frac{x}{2^{k}}\right)\right\|,\right. \\
& |2|^{k-1}\left\|(T-f)\left(\frac{-x}{2^{k}}\right)\right\|, \\
& |2|^{k-1}\left\|\left(f-T^{\prime}\right)\left(\frac{x}{2^{k}}\right)\right\| \text {, } \\
& |2|^{k-1}\left\|\left(f-T^{\prime}\right)\left(\frac{-x}{2^{k}}\right)\right\| \text {, } \\
& \frac{|a|^{2 k}}{|2|}\left\|(T-f)\left(\frac{x}{a^{k}}\right)\right\| \text {, } \\
& \frac{|a|^{2 k}}{|2|}\left\|(T-f)\left(\frac{-x}{a^{k}}\right)\right\|, \\
& \frac{|a|^{2 k}}{|2|}\left\|\left(f-T^{\prime}\right)\left(\frac{x}{a^{k}}\right)\right\|, \\
& \left.\frac{|a|^{2 k}}{|2|}\left\|\left(f-T^{\prime}\right)\left(\frac{-x}{a^{k}}\right)\right\|\right\} \\
& \leq \lim _{k \rightarrow \infty} \max \left\{\frac{|a|^{(2-r) k}\left(|a|^{r}+1\right) \theta\|x\|^{r}}{|2|^{2+r}|a|^{r}},\right. \\
& \left.\frac{|2|^{k(1-r)}\left(|a|^{r}+1\right) \theta\|x\|^{r}}{|2|^{2+r}|a|^{r}}\right\}=0,
\end{aligned}
$$

for all $x \in \mathscr{X}$, which means that $T$ is unique.

Theorem 8. Let $\varphi: \mathscr{X}^{2} \rightarrow[0, \infty)$ be a function such that

$$
\lim _{n \rightarrow \infty}|2|^{n} \varphi\left(\frac{x}{2^{n}}, \frac{y}{2^{n}}\right)=\lim _{n \rightarrow \infty} \frac{\varphi\left(a^{n} x, a^{n} y\right)}{|a|^{2 n}}=0,
$$


for all $x, y \in \mathscr{X}$ and, for each $x \in \mathscr{X}$, let the limit

$$
\begin{array}{r}
\lim _{n \rightarrow \infty} \max \left\{\frac{\varphi\left(0, a^{j} x\right)}{|2||a|^{2 j+2}},|2|^{j-1} \varphi\left(\frac{x}{2^{j+1}}, \frac{x}{2^{j+1} a}\right),\right. \\
\left.|2|^{j-1} \varphi\left(\frac{-x}{2^{j+1}}, \frac{-x}{2^{j+1} a}\right): 0 \leq j<n\right\},
\end{array}
$$

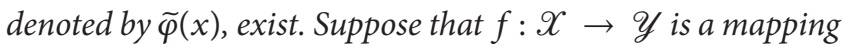
satisfying

$$
\|D f(x, y)\| \leq \varphi(x, y),
$$

for all $x, y \in \mathscr{X}$. Then there exists a quadratic-additive mapping $T: \mathscr{X} \rightarrow \mathscr{Y}$ such that

$$
\|f(x)-T(x)\| \leq \widetilde{\varphi}(x),
$$

for all $x \in \mathscr{X}$, where the mapping $T$ is given by

$$
\begin{aligned}
T(x)=\lim _{n \rightarrow \infty} & {\left[\frac{f\left(a^{n} x\right)+f\left(-a^{n} x\right)}{2 a^{2 n}}\right.} \\
& \left.+2^{n-1}\left(f\left(\frac{x}{2^{n}}\right)-f\left(\frac{-x}{2^{n}}\right)\right)\right],
\end{aligned}
$$

for all $x \in \mathscr{X}$.

Proof. For a given mapping $f: \mathscr{X} \rightarrow \mathcal{Y}$ and $n \in \mathbb{N}$, let $J_{n} f: \mathscr{X} \rightarrow \mathscr{Y}$ be a mapping defined by

$$
J_{n} f(x)=\frac{f\left(a^{n} x\right)+f\left(-a^{n} x\right)}{2 a^{2 n}}+2^{n-1}\left(f\left(\frac{x}{2^{n}}\right)-f\left(\frac{-x}{2^{n}}\right)\right),
$$

for all $x \in \mathscr{X}$. Now we note that $J_{0} f(x)=f(x)$ and

$$
\begin{aligned}
& \left\|J_{j} f(x)-J_{j+1} f(x)\right\| \\
& =\|-\frac{D f\left(0, a^{j} x\right)}{2 a^{2 j+2}}+2^{j-1} D f\left(\frac{x}{2^{j+1}}, \frac{x}{2^{j+1} a}\right) \\
& -\quad 2^{j-1} D f\left(\frac{-x}{2^{j+1}}, \frac{-x}{2^{j+1} a}\right) \| \\
& \leq \max \left\{\frac{\left\|D f\left(0, a^{j} x\right)\right\|}{|2||a|^{2 j+2}}, \| \frac{x}{|2|^{j-1}\left\|D f\left(\frac{x}{2^{j+1}}, \frac{x}{2^{j+1} a}\right)\right\|,}\right. \\
& \leq \max \left\{\frac{\varphi\left(0,\left.\right|^{j-1}\left\|D f\left(\frac{-x}{2^{j+1}}, \frac{-x}{2^{j+1} a}\right)\right\|\right\}}{|2||a|^{2 j+2}}, \ldots\right. \\
& |2|^{j-1} \varphi\left(\frac{x}{2^{j+1}}, \frac{x}{2^{j+1} a}\right), \\
& \left.|2|^{j-1} \varphi\left(\frac{-x}{2^{j+1}}, \frac{-x}{2^{j+1} a}\right)\right\},
\end{aligned}
$$

for all $x \in \mathscr{X}$ and $j \geq 0$. Hence it follows by (41) and (47) that the sequence $\left\{J_{n} f(x)\right\}$ is Cauchy. Since $\mathcal{Y}$ is complete, we conclude that $\left\{J_{n} f(x)\right\}$ is convergent. Thus we can define $T: X \rightarrow \mathscr{y}$ by

$$
T(x):=\lim _{n \rightarrow \infty} J_{n} f(x)
$$

for all $x \in \mathscr{X}$. Using the induction argument, we prove that

$$
\begin{aligned}
& \| J_{n} f(x)- f(x) \| \\
& \leq \max \left\{\frac{\varphi\left(0, a^{j} x\right)}{|2||a|^{2 j+2}},|2|^{j-1} \varphi\left(\frac{x}{2^{j+1}}, \frac{x}{2^{j+1} a}\right),\right. \\
&\left.|2|^{j-1} \varphi\left(\frac{-x}{2^{j+1}}, \frac{-x}{2^{j+1} a}\right): 0 \leq j<n\right\},
\end{aligned}
$$

for all $n \in \mathbb{N}$ and all $x \in \mathscr{X}$. Taking the limit as $n \rightarrow \infty$ in (49) and using (41) lead to inequality (44).

By virtue of (43), we get

$$
\begin{aligned}
\left\|D J_{n} f(x, y)\right\|= & \| \frac{D f\left(a^{n} x, a^{n} y\right)+D f\left(-a^{n} x,-a^{n} y\right)}{2 a^{2 n}} \\
& +2^{n-1}\left(D f\left(\frac{x}{2^{n}}, \frac{y}{2^{n}}\right)-D f\left(\frac{-x}{2^{n}}, \frac{-y}{2^{n}}\right)\right) \| \\
\leq & \max \left\{\frac{\varphi\left(a^{n} x, a^{n} y\right)}{|2||a|^{2 n}}, \frac{\varphi\left(-a^{n} x,-a^{n} y\right)}{|2||a|^{2 n}},\right. \\
|2|^{n-1} \varphi\left(\frac{x}{2^{n}}, \frac{y}{2^{n}}\right), & \left.|2|^{n-1} \varphi\left(\frac{-x}{2^{n}}, \frac{-y}{2^{n}}\right)\right\},
\end{aligned}
$$

for all $x, y \in \mathscr{X}$. Send the limit as $n \rightarrow \infty$ and then use (41) to find $D T(x, y)=0$ for all $x, y \in \mathscr{X}$.

Theorem 9. Let $\varphi: \mathscr{X}^{2} \rightarrow[0, \infty)$ be a function such that

$$
\lim _{n \rightarrow \infty} \frac{\varphi\left(2^{n} x, 2^{n} y\right)}{|2|^{n}}=\lim _{n \rightarrow \infty}|a|^{2 n} \varphi\left(\frac{x}{a^{n}}, \frac{y}{a^{n}}\right)=0,
$$

for all $x, y \in \mathscr{X}$ and, for each $x \in \mathscr{X}$, let the limit

$$
\begin{aligned}
\lim _{n \rightarrow \infty} \max \{ & \frac{|a|^{2 j}}{|2|} \varphi\left(0, \frac{x}{a^{j+1}}\right), \\
& \left.\frac{\varphi\left(2^{j} x, 2^{j} x / a\right)}{|2|^{j+2}}, \frac{\varphi\left(-2^{j} x,-2^{j} x / a\right)}{|2|^{j+2}}: 0 \leq j<n\right\},
\end{aligned}
$$

denoted by $\tilde{\varphi}(x)$, exist. Suppose that $f: \mathscr{X} \rightarrow \mathcal{Y}$ is a mapping satisfying

$$
\|D f(x, y)\| \leq \varphi(x, y)
$$


for all $x, y \in X$. Then there exists a quadratic-additive mapping $T: X \rightarrow Y$ such that

$$
\|f(x)-T(x)\| \leq \widetilde{\varphi}(x),
$$

for all $x \in \mathscr{X}$, where the mapping $T$ is given by

$$
\begin{array}{r}
T(x)=\lim _{n \rightarrow \infty}\left[\frac{a^{2 n}}{2}\left(f\left(\frac{x}{a^{n}}\right)+f\left(\frac{-x}{a^{n}}\right)\right)\right. \\
\left.+\frac{f\left(2^{n} x\right)-f\left(-2^{n} x\right)}{2^{n+1}}\right],
\end{array}
$$

for all $x \in \mathscr{X}$.

Proof. For a given mapping $f: \mathscr{X} \rightarrow \mathscr{Y}$ and $n \in \mathbb{N}$, let $J_{n} f: \mathscr{X} \rightarrow \mathscr{Y}$ be a mapping defined by

$$
J_{n} f(x)=\frac{a^{2 n}}{2}\left(f\left(\frac{x}{a^{n}}\right)+f\left(\frac{-x}{a^{n}}\right)\right)+\frac{f\left(2^{n} x\right)-f\left(-2^{n} x\right)}{2^{n+1}},
$$

for all $x \in \mathscr{X}$. We remark that $J_{0} f(x)=f(x)$ and

$$
\begin{aligned}
&\left\|J_{j} f(x)-J_{j+1} f(x)\right\|= \| \frac{a^{2 j}}{2} D f\left(0, \frac{x}{a^{j+1}}\right) \\
&-\frac{D f\left(2^{j} x, 2^{j} x / a\right)}{2^{j+2}} \\
&+\frac{D f\left(-2^{j} x,-2^{j} x / a\right)}{2^{j+2}} \| \\
& \leq \max \left\{\frac{|a|^{2 j}}{|2|}\left\|D f\left(0, \frac{x}{a^{j+1}}\right)\right\|,\right. \\
& \frac{\left\|D f\left(2^{j} x, 2^{j} x / a\right)\right\|}{|2|^{j+2}}, \\
& \leq \max \left\{\frac{|| D f\left(-2^{j} x,-2^{j} x / a\right) \|}{|2|^{j+2}} \varphi\left(0, \frac{x}{a^{j+1}}\right),\right. \\
& \frac{\varphi\left(2^{j} x, 2^{j} x / a\right)}{|2|^{j+2}}, \\
&\left.\frac{\varphi\left(-2^{j} x,-2^{j} x / a\right)}{|2|^{j+2}}\right\},
\end{aligned}
$$

for all $x \in \mathscr{X}$ and $j \geq 0$. So it follows from (51) and (57) that the sequence $\left\{J_{n} f(x)\right\}$ is Cauchy. Due to the completeness of $\mathscr{Y}$, this sequence is convergent. Let $T: \mathscr{X} \rightarrow \mathscr{Y}$ be a mapping defined by

$$
T(x):=\lim _{n \rightarrow \infty} J_{n} f(x),
$$

for all $x \in \mathscr{X}$. An induction implies that

$$
\begin{aligned}
\left\|J_{n} f(x)-f(x)\right\| \leq \max \left\{\frac{|a|^{2 j}}{|2|} \varphi\left(0, \frac{x}{a^{j+1}}\right),\right. & \frac{\varphi\left(2^{j} x, 2^{j} x / a\right)}{|2|^{j+2}}, \\
& \left.\frac{\varphi\left(-2^{j} x,-2^{j} x / a\right)}{|2|^{j+2}}: 0 \leq j<n\right\},
\end{aligned}
$$

for all $n \in \mathbb{N}$ and all $x \in \mathscr{X}$. By passing the limit as $n \rightarrow \infty$ in (59) with (51), we obtain the relation (54).

From (53), we find that

$$
\begin{array}{r}
\left\|D J_{n} f(x, y)\right\|=\| \frac{a^{2 n}}{2}\left(D f\left(\frac{x}{a^{n}}, \frac{y}{a^{n}}\right)+D f\left(\frac{-x}{a^{n}}, \frac{-y}{a^{n}}\right)\right) \\
+\frac{D f\left(2^{n} x, 2^{n} y\right)-2^{n} D f\left(-2^{n} x,-2^{n} y\right)}{2^{n+1}} \| \\
\leq \max \left\{\frac{|a|^{2 n}}{|2|} \varphi\left(\frac{x}{a^{n}}, \frac{y}{a^{n}}\right), \frac{|a|^{2 n}}{|2|} \varphi\left(\frac{-x}{a^{n}}, \frac{-y}{a^{n}}\right),\right. \\
\left.\frac{\varphi\left(2^{n} x, 2^{n} y\right)}{|2|^{n+1}}, \frac{\varphi\left(-2^{n} x,-2^{n} y\right)}{|2|^{n+1}}\right\},
\end{array}
$$

for all $x, y \in \mathscr{X}$. Taking the limit as $n \rightarrow \infty$ and using (51), we arrive at $D T(x, y)=0$ for all $x, y \in \mathscr{X}$.

Corollary 10. Let $|a|,|2|<1$ and let $r$ be a real number such that $1<r<2$. If a mapping $f: X \rightarrow \mathscr{Y}$ satisfies the condition

$$
\|D f(x, y)\| \leq \theta\left(\|x\|^{r}+\|y\|^{r}\right),
$$

for all $x, y \in \mathscr{X}$, then there exists a unique quadratic-additive mapping $T: X \rightarrow Y$ such that

$$
\|f(x)-T(x)\| \leq \frac{\left(|a|^{r}+1\right) \theta\|x\|^{r}}{|2|^{2}|a|^{r}},
$$

for all $x \in \mathscr{X}$.

Proof. We consider $\varphi(x, y)=\theta\left(\|x\|^{r}+\|y\|^{r}\right)$. Based on the fact that $|a|^{2-r}<1$ and $|2|^{r-1}<1$, the mapping $\varphi$ satisfies conditions (51). In fact, it is easy to see that

$$
\begin{aligned}
\widetilde{\varphi}(x) & =\max \left\{\frac{\theta\|x\|^{r}}{|2||a|^{r}} \frac{\left(|a|^{r}+1\right) \theta\|x\|^{r}}{|2|^{2}|a|^{r}}\right\} \\
& =\frac{\left(|a|^{r}+1\right) \theta\|x\|^{r}}{|2|^{2}|a|^{r}} .
\end{aligned}
$$

On account of Theorem 9, there is a quadratic-additive mapping $T: \mathscr{X} \rightarrow \mathscr{Y}$ satisfying (62). 
To show uniqueness of the mapping $T$, we suppose that $T$ is another quadratic-additive mapping satisfying (62). Then

$$
\begin{aligned}
& T^{\prime}(x)-J_{k} T^{\prime}(x) \\
& =\sum_{j=0}^{k-1}\left(\frac{a^{2 j}}{2} D f\left(0, \frac{x}{a^{j+1}}\right)\right. \\
& \left.\quad-\frac{D f\left(2^{j} x, 2^{j} x / a\right)}{2^{j+2}}+\frac{D f\left(-2^{j} x,-2^{j} x / a\right)}{2^{j+2}}\right)=0,
\end{aligned}
$$

for any $k \in \mathbb{N}$ and so we figure out the following:

$$
\begin{aligned}
& \left\|T(x)-T^{\prime}(x)\right\|=\lim _{k \rightarrow \infty}\left\|J_{k} T(x)-J_{k} T^{\prime}(x)\right\| \\
& \leq \lim _{k \rightarrow \infty} \max \left\{\left\|J_{k} T(x)-J_{k} f(x)\right\|,\right. \\
& \left.\left\|J_{k} f(x)-J_{k} T^{\prime}(x)\right\|\right\} \\
& \leq \lim _{k \rightarrow \infty} \max \left\{|2|^{-k-1}\left\|T\left(2^{k} x\right)-f\left(2^{k} x\right)\right\|,\right. \\
& |2|^{-k-1} \\
& \times\left\|T\left(-2^{k} x\right)-f\left(-2^{k} x\right)\right\|, \\
& |2|^{-k-1}\left\|f\left(2^{k} x\right)-T^{\prime}\left(2^{k} x\right)\right\|, \\
& |2|^{-k-1} \\
& \times\left\|f\left(-2^{k} x\right)-T^{\prime}\left(-2^{k} x\right)\right\|, \\
& \frac{|a|^{2 k}}{|2|}\left\|(T-f)\left(\frac{x}{a^{k}}\right)\right\| \text {, } \\
& \frac{|a|^{2 k}}{|2|}\left\|(T-f)\left(\frac{-x}{a^{k}}\right)\right\|, \\
& \frac{|a|^{2 k}}{|2|}\left\|\left(f-T^{\prime}\right)\left(\frac{x}{a^{k}}\right)\right\|, \\
& \left.\frac{|a|^{2 k}}{|2|}\left\|\left(f-T^{\prime}\right)\left(\frac{-x}{a^{k}}\right)\right\|\right\} \\
& \leq \lim _{k \rightarrow \infty} \max \left\{\frac{|a|^{k(2-r)}\left(|a|^{r}+1\right) \theta\|x\|^{r}}{|2|^{3}|a|^{r}},\right. \\
& \left.\frac{|2|^{k(r-1)}\left(|a|^{r}+1\right) \theta\|x\|^{r}}{|2|^{3}|a|^{r}}\right\} \\
& =0 \text {, }
\end{aligned}
$$

for all $x \in \mathscr{X}$. This implies that $T$ is unique.

The problem is whether or not the previous corollaries hold the cases when $r=1$ or $r=2$.

\section{Conflict of Interests}

The authors declare that there is no conflict of interests regarding the publication of this paper.

\section{Acknowledgments}

The authors would like to thank the referees for giving useful suggestions and for the improvement of this paper. The first author was supported by Basic Science Research Program through the National Research Foundation of Korea (NRF) funded by the Ministry of Education (no. 2013R1A1A2A10004419).

\section{References}

[1] S. M. Ulam, A Collection of the Mathematical Problems, Interscience, New York, NY, USA, 1960.

[2] D. H. Hyers, "On the stability of the linear functional equation," The Proceedings of the National Academy of Sciences of the United States of America, vol. 27, pp. 222-224, 1941.

[3] T. Aoki, "On the stability of the linear transformation in Banach spaces," Journal of the Mathematical Society of Japan, vol. 2, pp. 64-66, 1950.

[4] T. M. Rassias, "On the stability of the linear mapping in Banach spaces," Proceedings of the American Mathematical Society, vol. 72, pp. 297-300, 1978 .

[5] N. Brillouet-Belluot, J. Brzdęk, K. Cieplinski, and J. Szczawiriska, "On some recent developments in Ulam's type stability," Abstract and Applied Analysis, vol. 2012, Article ID 716936, 41 pages, 2012.

[6] K. Cieplinski, "Stability of multi-Jensen mappings in nonArchimedean normed spaces," in Fuctional Equations in Mathematical Anaysis, T. M. Rassias and J. Brzdęk, Eds., vol. 52 of Springer Optimization and Its Applications, pp. 79-86, Springer, New York, NY, USA, 2012.

[7] P. Gavruta, "A generalization of the Hyers-Ulam-Rassias stability of approximately additive mappings," Journal of Mathematical Analysis and Applications, vol. 184, pp. 431-436, 1994.

[8] S. Jung, On the Hyers-Ulam-Rassias Stability of Functional Equation in Nonlinear Analysis, Springer, New York, NY, USA, 2011.

[9] A. Najati and M. B. Moghimi, "Stability of a functional equation deriving from quadratic and additive functions in quasi-Banach spaces," Journal of Mathematical Analysis and Applications, vol. 337, no. 1, pp. 399-415, 2008.

[10] T. Z. Xu, "On the stability of multi-Jesen mappings in nonArchimedean normed spaces," Journal of Mathematical Physics, vol. 25, Article ID 023507, 9 pages, 2012.

[11] T. Z. Xu, J. M. Rassias, and W. X. Xu, "A fixed point approach to the stability of a general mixed AQCQ-functional equation in non-archimedean normed spaces," Discrete Dynamics in Nature and Society, vol. 2010, Article ID 812545, 24 pages, 2010.

[12] J. Brzdęk and K. Ciepliński, "A fixed point approach to the stability of functional equations in non-Archimedean metric spaces," Nonlinear Analysis, Theory, Methods and Applications, vol. 74, no. 18, pp. 6861-6867, 2011.

[13] J. Brzdęk and K. Cieplinski, "A fixed point theorem and the Hyers-Ulam stability in non- Archimedean spaces," Journal of Mathematical Analysis and Applications, vol. 400, pp. 68-75, 2013. 
[14] M. S. Moslehian and T. M. Rassias, "Stability of functional equations in non-archimedean spaces," Applicable Analysis and Discrete Mathematics, vol. 1, no. 2, pp. 325-334, 2007.

[15] A. Bahyrycz, J. Brzdęk, and M. Piszczek :, "On approximately p-Wright affine functions in ultra metric spaces," Journal of Function Spaces and Applications, vol. 2013, Article ID 723545, 7 pages, 2013.

[16] Y. Lee, "Hyers-Ulam-Rassias stability of a quadratic-additive type functional equation on a restricted domain," International Journal of Mathematical Analysis, vol. 7, pp. 2745-2752, 2013.

[17] M. Piszczek and J. Szczawiska, "Hyperstability of the Drygas functional equations," Journal of Function Spaces and Applications, vol. 2013, Article ID 912718, 4 pages, 2013. 


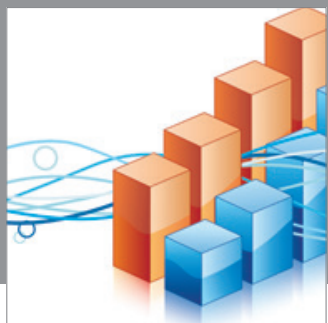

Advances in

Operations Research

mansans

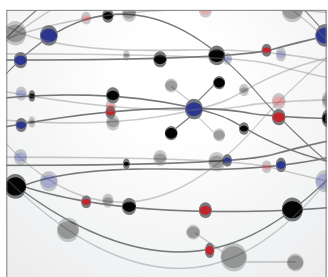

The Scientific World Journal
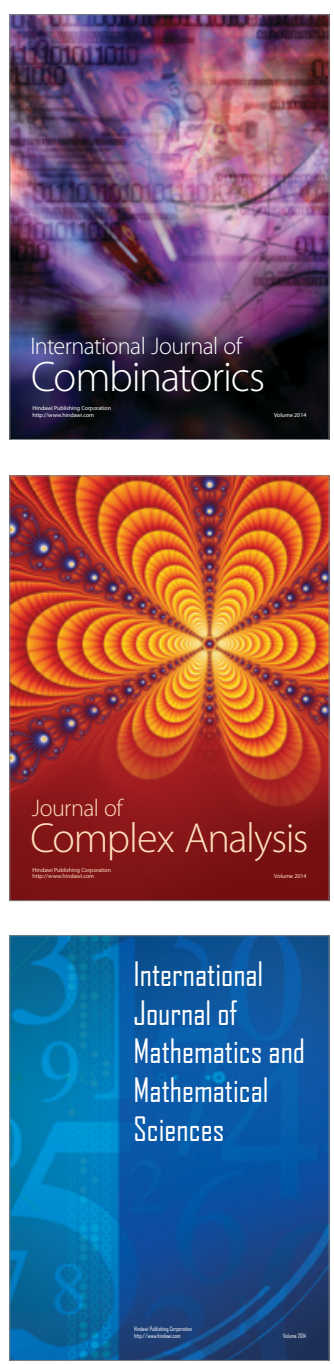
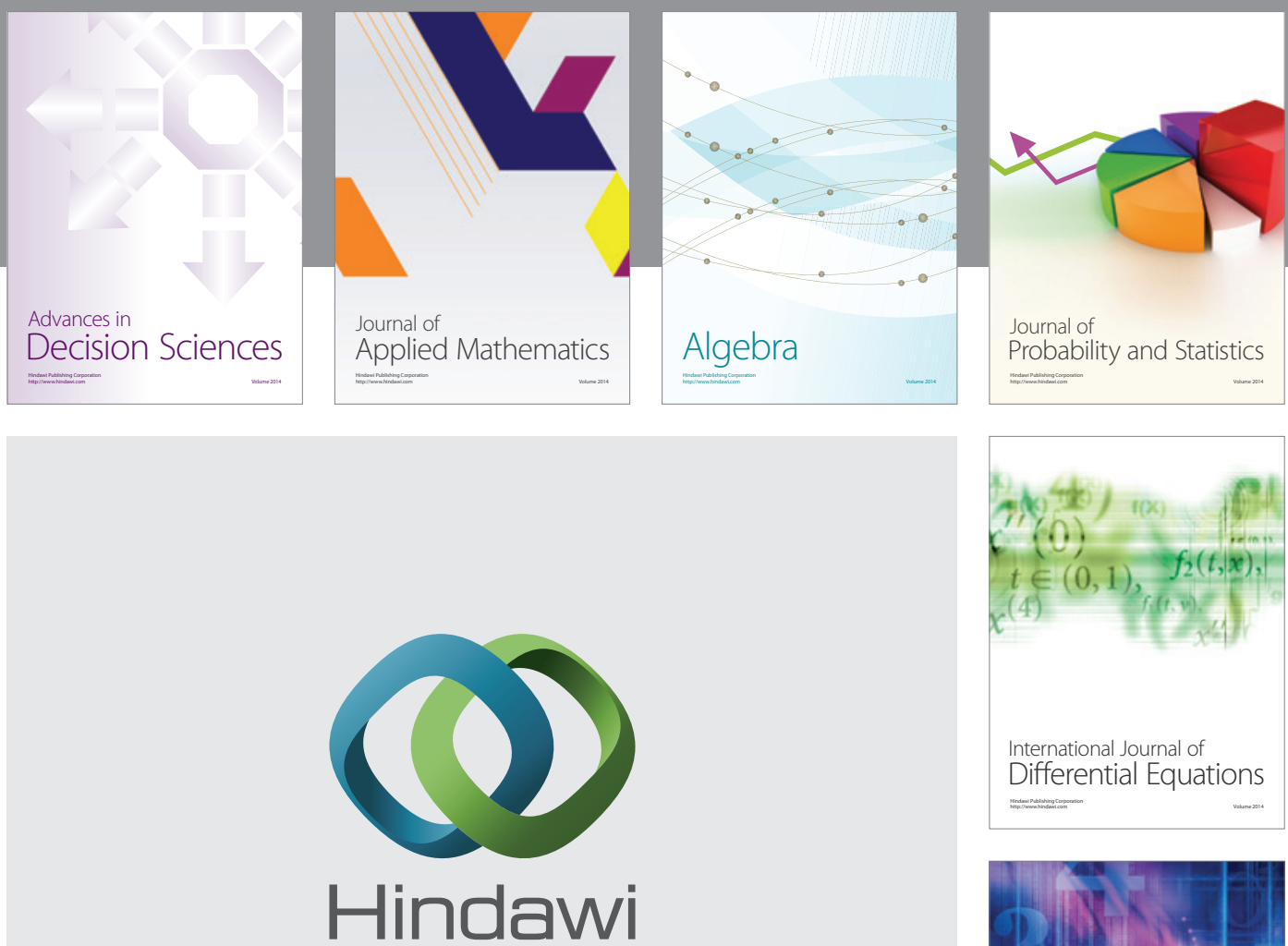

Submit your manuscripts at http://www.hindawi.com
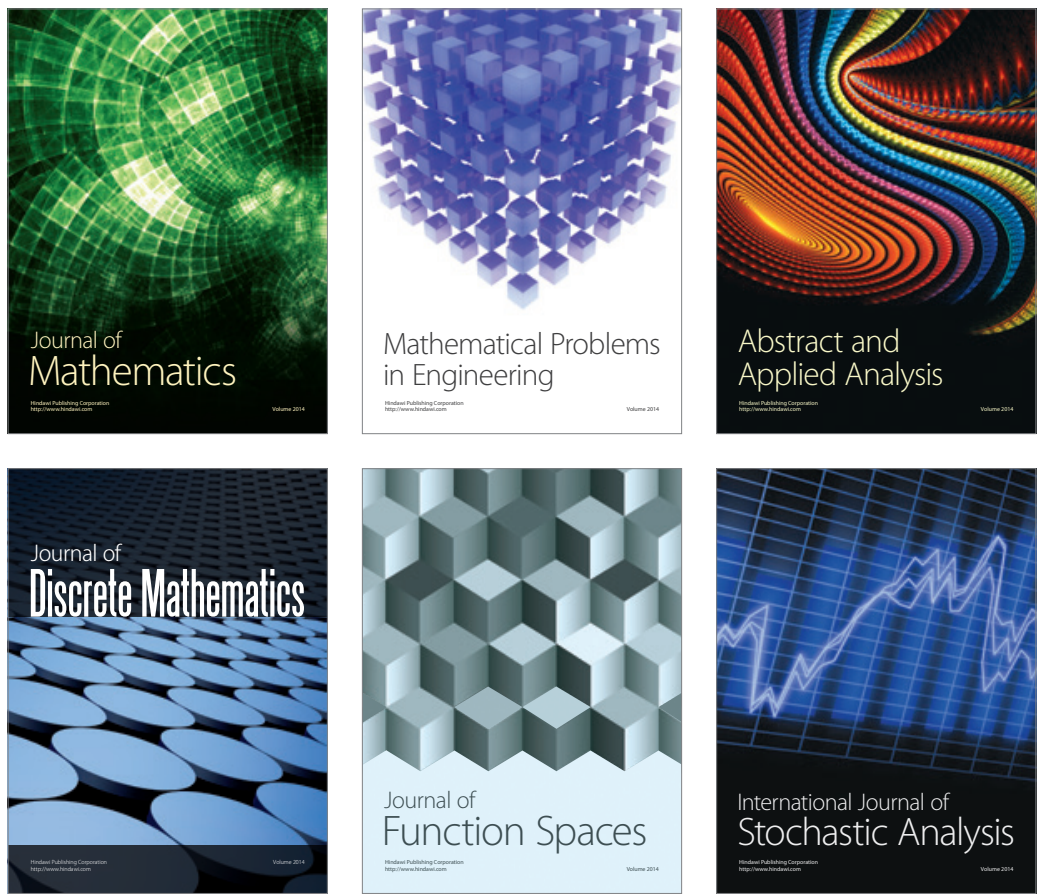

Journal of

Function Spaces

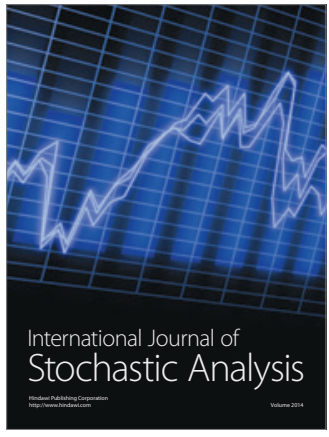

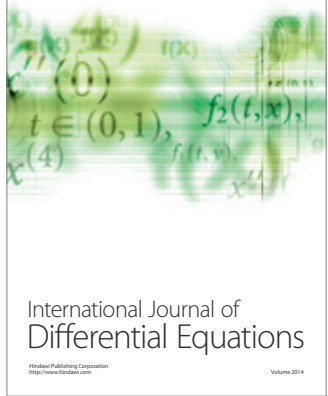
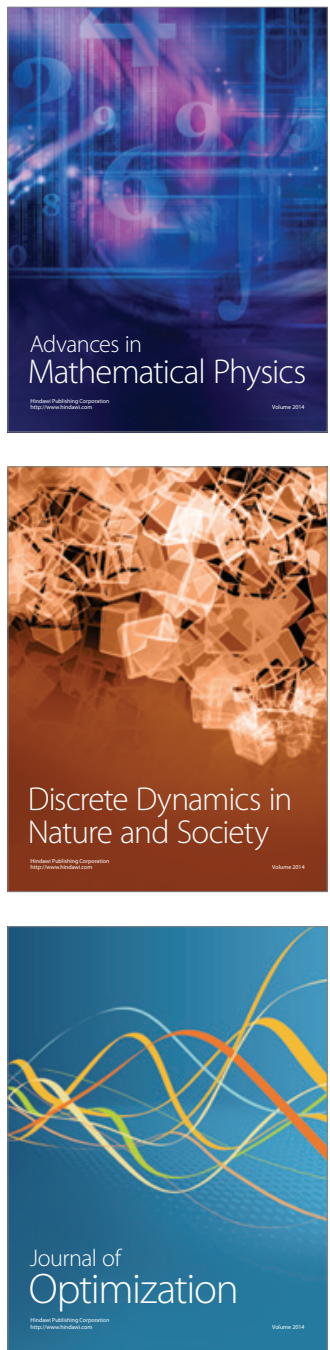